profils en long du Verdon, qui ont été levés, l'un en 1878 , l'autre en $x 899$, nous avons pu calculer aussi exactement que possible le cube de gravier qui correspond à une période de . ans. Il en résulte que, en moyenne, le Verdon ne charrie que 50.000 $\mathrm{m}^{3}$ de gravier par an.

Comme le barrage de Quinson retiendra les graviers pendant encore fort longtemps, une centaine d'années probablement, on voit qu'il n'y a pas à se préoccuper de cette question pour le réservoir de Gréoulx.

Le Verdon est, il est vrai, la seule rivière pour laquelle il nous a été possible de déterminer par un mesurage direct le cube de gravier cntrainé. Mais, en nous basant sur certaines observations, nous avons établi une méthode de calcul qui peut domer d'une façon plus ou moins approximative le cube du gravier charrié par d'autres rivières torrentielles.

Si le cube du gravier est relativement peu important, cehi du limon tenu en suspension dans l'eau est, par conLre, très grand. Ce cube peut être déterminé assez exactcment on prenant tous les jours un ćchantillon d'eau, et en pesant te limon qu'il contient après l'avoir recueilli par décantition et filtration. Connaissant les débits journaliers de la rivière pendant une certaine période, on en déduira ensuile lo cube de limon qu'elle aura transporté pendant le même temps.

Cette méthode a été appliquée à la Durance à Mirabeau, pendant un grand nombre d'années, par Hervé-Mangon. Elle a permis d'évaluer à cinq millions de mètres cubes environ le volume moyen du limon charrié annuellement par la Durance en ce point. Par comparaison, on peut estimex ì $700.000 \mathrm{~m}^{3}$ le cube correspondant pour le Verdon à Quinson.

Ces chiffres montrent la nécessité d'évacuer le limon qui se déposera dans le réservoir de Gréoulx. En négligeant de le faire, la perte de capacité utile atteindrait environ sept millions de mètres cubes en dix ans, et trente-cinq millions en cinquante ans. Nous pensons que pour arriver à un dévasement efficace de ce réservoir, il ne sera pas suffisant d'employer les moyens qui ont été usités jusqu'à présent.

Le procédé classique, qui fonctionne avec succès en Espagne et en Algérie, consiste à ouvrir brusquement des évacuatcurs de fond lorsqu'il n'y a plus qu'une faible hauteur d'eau dans le réservoir. Cette opération, combinée avec l'action des crues, est répétée un certain nombre de fois. Pour obtenir de bons résultats, il faut que le cours d'eau sur lequel est construit le barrage soit bien encaissé, et que la longueur du lac artificiel dans lequel se dépose le limon ne soit pas trop grande. Dans un réservoir de l'importance de celui de Gréoulx, il est de toute évidence que ce procédé ne donnerait pas des résultats suffisants, car l'appel des évacuateurs ne pourrait se faire sentir que dans une faible partie de la retenue et, d'autre part, l'action des crues dans le réservoir supposé vide serait peu efficace dans les parties où celui-ci comportera un fond large et plat, c'est-à-dire dans celles où la capacité utile sera la plus grande.

Pour compléter l'action des évacuateurs, il sera indispensable de recourir à des procédés mécaniques. On pourra,dans ce but, employer de puissantes dragues suceuses qui aspireront les vases, après les avoir soulevées par l'injection d'eau comprimée, et qui les refouleront de proche en proche, au moyen de baches flottantes, dans la zone d'aclion des évacuateurs. Ces dragues pourront être actionnées électriquement par la force motrice de la chute du barrage.

Fonctionnement de la retenue. - Le réservoir de Gréoulx pourra emmagasiner un volume d'eau de 97 millions de mètres cubes, qui sera largement suffisant pour faire face à une pénurie moyenne, et qui permettra de remédier presque complètement à une pénurie exceptionnelle, telle que celles de $\mathrm{x} 896$ et $\mathbf{g} g 06$.

Le calcul des débits du Verdon, que nous avons pu faire avec une assez grande précision pour une période de vingt ans, nous a permis de conclure que l'alimentation du réservoir sera loujours bien assurée, même dans les anmécs les plus sèches. On peut donc compler que celui-ci sera toujours plein au $1^{\text {er }}$ aoùt, c'est-ì-dire avant le commencenent de la pénuric d'cau.

Avec un cube ulile qui ne descendia jamais en-dessous de go millions de mères cubes, on pourra augmenter le débit du Verdon, et pal suite celui de la Durance :

$\begin{array}{ccccc}\text { de } & 17 & 26 & 34 & \mathrm{~m}^{3} \\ \text { pendant } & 60 & 40 & 30 & \text { jours }\end{array}$

Dépense. - La dépense du barrage-réservoir de Ciréoulx s'élève à douze millions de franes, dont la moilié sera supportée, comme nous l'avons expliqué, par Jes départements des Bouches-du-Rhóne el de Vaucluse. En présence de celle contribution très importanie, consentie par les intéressés, il parait probable que le Parlement n'hésitera pas ì déclarer d'utilité publique l'exéculion, par l'Etat, des travaux du barrage-réservoir de Grćoulx.

Un projel de loi a été déposé dans ce but au mois d'avril dernier, mais il n'a pu être disculé arant la fin de la légism lature.

(1 suivre.)

I. WILHein.

Ingónieur en Chef des Ponls-el-Chuussées.

\section{NOUVELLE MÉTHODE DE JAUGEAGE PAR FLOTTEURS}

Il existe, dans l'étal actuel de l'Hydraulique, un certain nombre de méthodes qui permettent de déterminer approximativement le débit d'un cours d'eau. Mais on conviendra qu'elles sont, pour la plupart, d'une application malaisêe: elles exigent une mise en scène importante, un matériel de mesure onéreux, un doigté expérimental qui ne peut être que le privilège du spćcialiste. Aussi, n'a-t-on recour's à ces méthodes que dans d'exceptionnelles circonslances, lorsque la chute est d'assez grosse puissance pour justifier une sérieuse étude préalable, et la contribution de l'ingénieur hydraulicien.

Mais, bien souvent, la chute ne mérite pas qu'on se mette en frais. Et le débit en est déterminé suivant des procédés empiriques, issus guelquefois d'une lointaine tradition. C'est le cas, si fréquent, des petites chutes de montagrie; les indigènes les aménagent eux-mêmes, plus confiants en la mode du pays qu'en la science suspecte et couteuse de l'ingénieur. C'est dire quils gaspillent en pure perte l'eau du torrent dont ils méconnaissent la puissance latente, et leurs installations invraisemblables n'ont jamais qu'un rendement déplorable.

En attendant que les stalions centrales aient étendu leur emprise jusque dans ces petites vallees, il no paralt pas inutile de venir en aide à l'ingéniosité simpliste de leurs populations. Celles-ci, d'ailleurs, prises d'une récente émulation, sont disposées à sacrifier leurs receltes seculaires, et à adopter des procédés plus techniques, propres à assurer une meilleure utilisation de leurs chutes. Encore fautil que cus procédés leur soient d'une compréhension simple et d'une exécution facile. 
C'est dans cette unique pensée d'assistance technique que nous croyons devoir présenter une méthode de détermination des débits, encore inédile, croyons nous. Elle est d'une extrême simplicité, et il nous semble qu'elle pourrait être d'une utile contribution à laménagement des cliutes de montagne.

Cette méthode nous a été sugrérée dans des circonstances assez pittoresques pour mériter d'être citées.

Il existe, en haute terre ariégeoise, un grand nombre do bourgades qui détiennent leur prospérité de la seule initialive privée. Toutes les chutes, si minimes soient-elles, échelonnées sur le parcours de la Thuys, affluent capricieux de l'Ariège, ont été asservies par les indigènes. La force extraite du flot est une ressourco si précieuse pour la mise en ceuvre des petites industries locales, qu'en plusieurs endroits les riverains so partagent jalousement l'eau du torrent.

En amont des prises d'eau, sur une longueur d'environ dix mètres, une cloison formée de l'assemblage de quelques poutres est établie sur le milieu du lit, à égalo distance des deux rives. La rivière se trouve ainsi diviséc en deux bras d'égale section. Mais ceci ne suffit pas à leur assurer le mème débit, car lune des voles peut avoir une vitesse decoulement plus grande que lautre. Les propriétaires, interesses jusqu'à prétendre à un partage rigoureux, decelent cette difference, que le seul aspect des nappes superficielles ne suffit pas à rendre appréciable, au moyen de lexprience plus probante que voici.

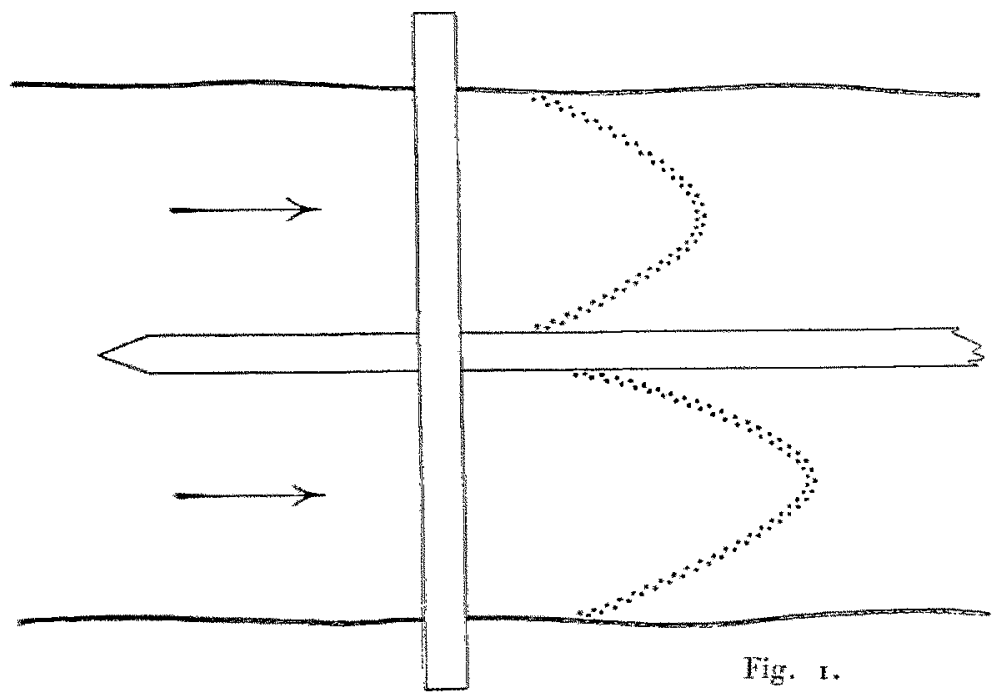

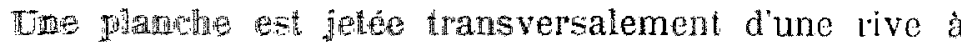

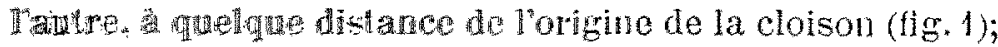

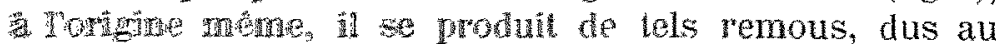

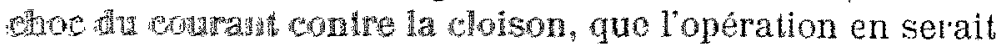

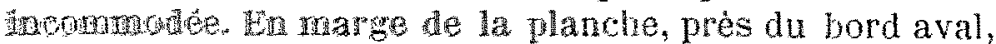
Ton dispose wre mince langeg de confotis.

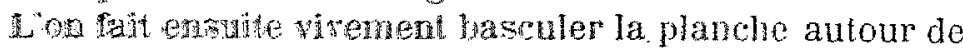
se bord. Les ondertus lomben a la surface de l'eau;

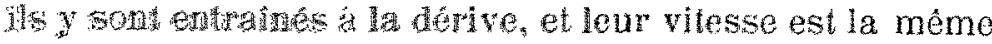
due entex des fllets qui les vehiculent. C'est dire que dons ougune des voles deau, ils vont plus vite au milea gue pros aes londs. lis y dessinent, apres quelques secondes. upe compe monvante dont la convexite va saccentuant Mats a méne instant, on pout considerer les

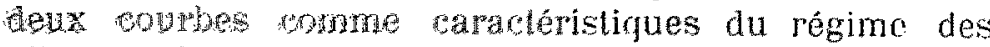
witesses dans les aenz voies deau. on peut meme

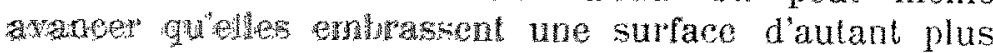

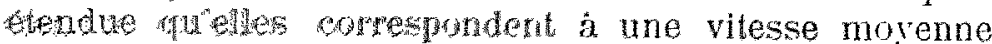

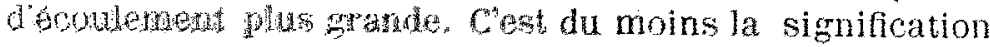

que leur attribuent, pour les besoins de la cause, ces hydrauliciens improvisês de. la montagne. De l'inégalité apparente dos surfaces comprises entre lo bor aval de la planche et chacune des courbes figurées par les confettis, ils concluent à une inégalité de même sens des débits; et cette conclusion a toute chance, en général, d'ètre vraie, malgré que l'expérience des confettis ne se rapporte en réalité qu'aux nappos superficielles.

Pour corriger la difference ainsi rendue sensible, le riverain le moins favorisé creuse le lit de son canal, de manière à lui donner, à partir du seuil, une plus grande déclivité; ce qui a pour effet sans doute d'accroître la vitesso d'écoulement de leau, mais aussi de réduire la hauleur disponible de chute. Il n'empeche que le partage ost considéré comme définitif lorsque l'épreuve des confeltis, renouvelée, donne liou à des courbes aussi semblables que possible de forme ot d'étendue.

Si le lit de la riviere est constitué par une roche trop dure pour ètre entaillee, on procède autrement. On agit directement sur l'orientation du courant. En amont immédiat de la région de partage, on etablit contro les rives des blocs de maconnerio, ou plus simplement des monceaux de roche. Ces obstacles, convenablement disposcs, déterminent des reflux assez forts pour clésorienter le cours primitil de l'eau. On règle leur effet jusqu'á ce que le débit de la rivière so distribue également de part et d'autre de la cloison.... au dire des confettis.

Celle expérienco des confellis, d'uno simplicité si expres. sive, méritait d'être reprise sous une forme plus systémalique, se prêtant à la mosuro elle-même des débits.

Nous avons dil que la courhe dessinée par les confettis à un instant donnè est caractéristique du régime transversal des vitesses. Malheureusement, cette courbe se déforme, et il est matériellement impossible de la fixer. D'ailleurs, les confettis sont plus ou moins épars, et il serait difficile de décrire leur trajectoiro moyenne. Enfin, celte courbe n'exprime jamais que le régime des vitesses superficielles de l'eau.

Il importait done de lui substituer une courbe stable et linéaire, que l'on puisse relever sans difficultó el avec exactitude. Il importait aussi d'imaginer un mode opératoire faisant intervenir l'ensemble des vitosses de tous les filets de la rivièro. La méthodequi suit semble répondre à ces conäitions.

Principe de la méthode - Nous évaluerons le débit de la rivière en le considérant comme égal au produit de la section $H L$ du courant par sa vitesse moyenne $U$

La méthode consiste à déterminer celte vitesse mojenne en combinant la méthode ordinaire du flotteur avec une méthode funiculaire dérivée de l'expérience rudimentaire des confeltis.

La méthode du flotieur donne la vitesse moyenine d'une tranche verticale du courant. La méthode funiculaire, exposée ci-après, donne d'autre part la vitesse moyenne des tranches horizontales. En les complétant l'une par l'autre, on conçoit qu'on puisse obtenir une approximation supérieure.

\section{MÉTHODE FUNIGULAIRE}

Proposons-nous d'abord de déterminer la vi tesse moyenne do leau dans une seule nappe d'épaisseur tres petite paral. léle a la surface libre. Nous chercherons ensuite a totaliser les vitesses inoyennes des diflérentes nappes de manière a en déduire la vitesse moyenne globale du courant. 
Nous admottons que, dans la rérion d'essai, l'eau se déplace parallelement aux rives, el sans accélération sensible.

Au niveau de la nappe, dont il s'agit de mesurer la vitesso moyenne, nous fixons aux rives los extrémilós d'un fil fin homogene et parfaitement souple (*), dont la longueur soit plus grande que la largeur $L$ de la riviore. La ligno droite passant par les points d'attache doit être normale à la direction d'écoulement.

Le fil, chtrainé par le courant, se tend of se dispose en équilibro dans le plan de la nappe. Il y dessine une courbe plus ou :noins dissymétrique dont la forme est évidemment fonction du régime transversal des vitesses pour la nappe considerée. On conçit, en conséquence, quil soit possible d'en déduire la vilesse moyenne.

Relation entre la forme du fil et le régime transversal des vitesses. - Nous allons ćtablir quill existe une relation simplo entro la forme de la courbe el lo régime des vilesses.

L'expérience montre que le fil se lond à peu près hori. zonlalement sous l'action entrainante du courant. Tout se passe comme si son poids était exactement écuilibré par la ruaction verticale do l'eau; et l'on peut dire que la forms: d'éfuilibre du fil dépend de la seule poussée horizontale du courant. La poussée qui s'exerce en chaque point du fil est, cvidemment, une force parallè a a la direction du courant, et d'aulant plus intense que la vitesse de l'eau au point considéré est plus grande.

Definissons celte force. Pour cela, nous subdivisons la nappe agissant sur le fil en filets infiniment minces, parallèlos à la direction du courant.

Considérons l'un quelconque $\mathrm{F}$ de ces nlets frappant un élé. ment de fil incliné sur sa direction (fig. 2). Par suite de cette inclinaison, l'eau du flot tend à glisser suivant MG, le long du fil. Mais ce glissement

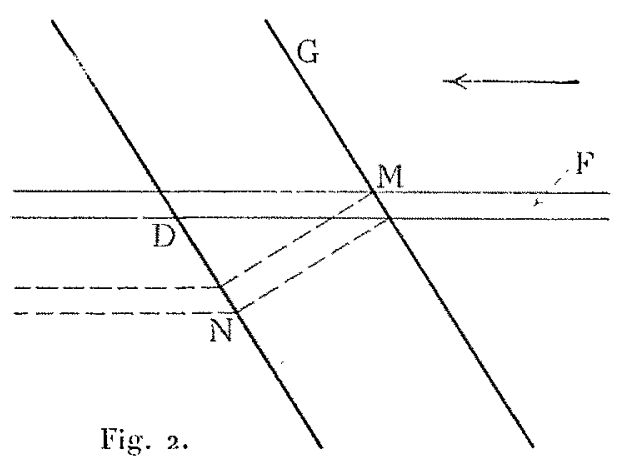

ne peut se produire: car le fil étant continu, tous les filets cui le rencontrent s'opposent mutuellement au glissement tangentiel de leurs molécules. Celles-ci n'ont d'autre issue que de contourner le fil par dessus et par dessous, et elles tendent évidemment à le rranchir par la voio la plus courte, c'est-à-dire en suivant la circonférence de section droite $\mathrm{MN}$.

En définitive, le filet considèré élant sollicité d'une part de glissor suivant MG el d'autre part de se dévier suivant MN, doit contourner le fil suivant une direction intermediaire et, vraisemblablement, suivant sa propre direction MD. Il suffit d'ailleurs d'observer l'aspect de l'eau incidente pour constater qu'il on est bien ainsi.

Dès lors, l'impulsion f exercée sur le fil est aussi dirigée suivant la direction naturelle du filet, quelle que soit d'ailleurs l'inclinaison du fil.

Cherchons la relation de grandeur existant entre celle impulsion et la vitesse. Pour cela, nous subdivisons la nappe leau agissant sur le fil en filcts infiniment minces. Quel que soit le mode d'écoulement dos molćcules lirui-

(*) Un til à coudre ordinaire salishat parfatement a ces condilions. des (mouvement toublillonnaire ou translation plus ou moins vagabonde a travers la masse deau mouvante), nous supposons que loutes les molécules liquides comprises, a un instant domné, dans une meme section droite d'un filet ont, suivant ce flet, une vitesse movenne de translation constante. Celte vitesse caractérislique de chaque filet varie d'ailleurs de l'un à l'autre suivant une loi conlinue.

Lexpression de la force $f$, en fonction de la vitesse $r$, ainsi définie, s'obtient en appliquant à l'élément de fil frappé par le filet $F$ la formule hien connue qui donne l'action oxercée par un liquide en translation rectiligne sur un corps immergé $\left(^{*}\right)$ :

$$
f=k s v^{2}
$$

dans laquelle $v$ est la vitesse du liquide, $s$ la projection du corps sur un plan normal à la direction d'ecoulement, et $k$ un coefficient qui dépend de la forme du corps immorgé

Nous aurons donc ici, en désignant par $d$ lo liamètre du fil, et par a l'épaisseur du filet considéré :

$$
f=k d \equiv v^{2}
$$

Le chemin curvilique MD suivi par les divers filets à la surface du fil diminue de longueur depuis les points d'attache du fil jusquien son milieu, of il semble que le coefficient $h$ doit varier un peu le long du fll. En réalité les éléments percutés par des filets d'égale largeur offrent au glissement superficiel des molécules liquides des surfaces de contact équivalentes. Aussi peut-on admettro que la variation possible du coefficient $k$ est infime, et nous le considérerons comme constant sur toute la longueur du fil.

Ceci posé, on peut faire ahstraction du mouvement ambiant de l'eau, el considérer le fil comme en équilibre statique dans un milieu immobile, sous la scule aclion des forces $f$ équivalentes à ce mouvemont. Formulons la loi d'équilibre dans de telles condilions.

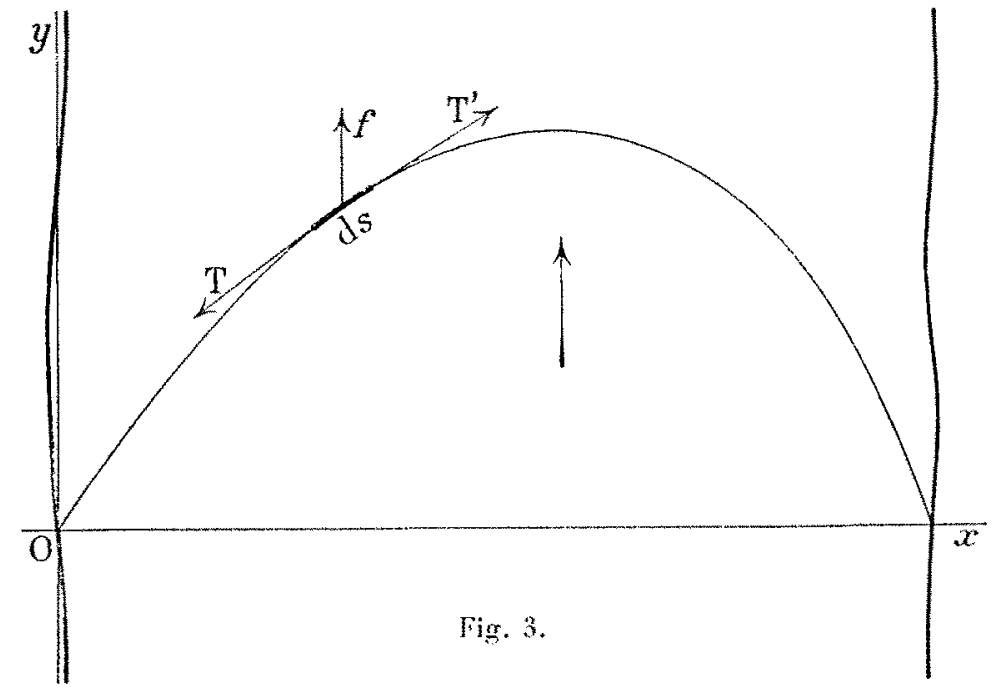

Rapportons la coupbe d'equilibre du fll a deux axes de coordonndes pris dans la nappe, lun ox passant pal les points d'attache, l'autre oy mené perpendiculairement a Ox dans le sens de l'écoulement do l'eau (fig. 3).

Considérons l'ólément de fil de longueur ds, percuté par le fllet liquide de vitesse $v$ comme matériellement indépendant du reste du fil. Cet elément est en équilibre autonome

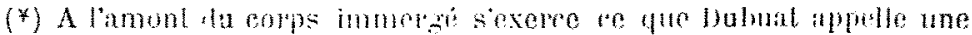

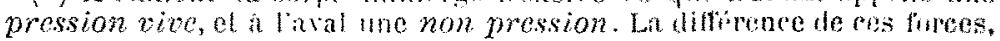
projelles stu lit dirolion du monrement, ast proporlionnelle an carré de lit vilesse dil fluide el a la seetion du contour apparent du copps immergé. 
sous l'action: to $^{\circ}$ de la force $f$, et $2^{\circ}$ des deux forces de tension $T$ et $T$ ' appliquées tangentiellement aux extrémités de l'élément, et tenant lieu de la liaison matérielle do l'élémont aveo le reste du fil.

La somme algúbrique de la projection de ces forces sur chacun des axes doil etre nulle. On aura donc:

sur $0 x:-T \frac{\mathrm{d} x}{\mathrm{~d} s}+\left[T \frac{\mathrm{d} x}{\mathrm{~d} s}+\mathrm{d}\left(T \frac{\mathrm{d} x}{\mathrm{~d} s}\right)\right]=0$

équation qui se roduit à :

$$
\mathrm{d}\left(T \frac{\mathrm{d} x}{\mathrm{~d} s}\right)=0
$$

sur oy: $\quad f-T \frac{\mathrm{d} y}{\mathrm{~d} s}+\left[T \frac{\mathrm{d} y}{\mathrm{~d} s}+\mathrm{d}\left(T \frac{\mathrm{d} y}{\mathrm{~d} s}\right)\right]=0$

équation çui se réduit à :

$$
f+\mathrm{d}\left(T \frac{\mathrm{d} y}{\mathrm{~d} s}\right)=0
$$

ou, en tenant compte que $f=k \cdot l^{2} \mathrm{~d} x$,

$$
k d v^{2} \mathrm{~d} x+\mathrm{d}\left(T \frac{\mathrm{d} y}{\mathrm{~d} s}\right)=0
$$

Eliminons entro les équations de projection (2) et (3) In tension inconnue $T$. Nous aurons une équation différenlielle en $x$ et $y$ qui sera l'équation d'équilibre du fil.

De l'équation (2) on tire :

$$
T \frac{\mathrm{d} x}{\mathrm{~d} s}=c
$$

$c$ étant une constante d'intégrration positive,

et de là : $\quad T=c \frac{\mathrm{d} s}{\mathrm{~d} \cdot \mathrm{x}}$

Substituant dans (3), il vient:

$$
k a v^{2} \mathrm{~d} x+\mathrm{d}\left(c \frac{\mathrm{d} s}{\mathrm{~d} x} \cdot \frac{\mathrm{d} y}{\mathrm{~d} s}\right)=0
$$

relation que nous écrirons :

$$
v^{2}+\frac{c}{h d} \cdot \frac{\mathrm{d}}{\mathrm{d} x}\left(\frac{\mathrm{d} y}{\mathrm{~d} x}\right)=0
$$

ou, plus simplement:

$$
\begin{gathered}
\frac{\mathrm{d}^{2} y}{\mathrm{~d} x^{2}}=-\left(\frac{v}{K}\right)^{2}, \\
K^{2}=\frac{c}{h d}
\end{gathered}
$$

en posant :

T'elle est l'équation dimerentielle remarquablement simple qui relie la configuration du fil au régime des vitesses.

Si lon convient de définir, en première analyse, le degré de concavité de la courbe en un point far la valeur de la dérivée seconde $\frac{\mathrm{d}^{2} y}{\mathrm{~d}^{2} x}$ on voit:

10 Qu'en valeur absolue, le derré de concavité cst proportionnel au carré de la vitesse d'écoulement en chaque point. C'est dire que la courbe sera sensiblement rectiligne dans les zones de vitesse très petite; elle présentera au contraire son maximum de courbure dans la zone la plus rapide. C'est co que vérifie lexpérience. Près des rives, ou l'eau se meut lentement, lo fil présente deux brins a peu prés roctilignes, au lieu qu'il est le plus incurve au milieu meme du courant:

$2^{\circ}$ Qu'en valeur algébrique le degré de concavité est constamment de même signe. C'est dire que la courbe tourne en tout point sa concavité en sens inverse du courant, sans inflexion possible $\left(^{*}\right)$.

Pour un courant idéal, dont la vitesse d'écoulement serait la même dans toute l'étendue de la nappe, tous les filels exeirceraient sur le fil la même action $f$, et l'équation diffé. rentielle de la courbe serait:

$$
\frac{\mathrm{d}^{2} y}{\mathrm{~d} x^{2}}=\text { constante }
$$

léquation intégrale serait de la forme:

$$
y=A x^{2}+B x \text {. }
$$

On reconnait l'équation d'une parabole, d'axe parallèle à $O y(\star \star)$.

Dans le cas d'un courant à vitesse lente, et par conséquent à peu près uniforme, l'expérience montre que la courbe du fil est effectivement une parabole légèrement déformèe.

Détermination graphique de la vitesse moyenne. Elant donnée la relation différentielle (4), il reste à en déduira la vitesse moyenne de la nappe :

$$
u=\frac{1}{L} \int_{0}^{L} v \mathrm{~d} x
$$

Supposons qu'on ait relevẻ expérimentalement la courbe d'équilibre $\mathrm{Y}$ du fil (fig. 3). Comme on ne peut évidemment expliciter sous forme algébrique la fonction $y$ de $a$ représentée par cette courbe, la relation (4) ne peut permettre de formuler analy tiquement $v$ en fonction do $x$, et le calcul de la vitesse moyenne par intégration n'est pas possible.

Il n'est heureusement pas indispensable d'avoir recours à la détermination algébrique de la fonction $y$; il suffit d'opérer sur la représentation graphique elle-mème. Voici comment.

Il est facile de déduire de la courbe Y la courbe dérivée première $Y$ ' représentant en fonction de $x$ le coefficient angulaire $\frac{\mathrm{d} y}{\mathrm{~d} x}$ de la tangente à $\mathrm{Y}$. II suffit d'employer le dispositif graphique suivant (fig. 4), qui permet de passer rapidement des différents points de $Y$ aux points homologues de Y'.

Portons sur AB un segment AP représentant. à l'échelle des coordonnées, lunité de longueur. Pour transformer un point $\mathrm{M}$ de $\mathrm{Y}$, il suffit de mener la parallèle $\mathrm{AM}_{1}$ à la tangente MT, dont le coefficient angulaire est ainsi représenté par le segment $\mathrm{PM}_{1}$; en reportant ce segment sur l'ordonnée du point $M$, on $a$, en position, l'homologue $M$ ' de $M$.

La position du segment unité peut être quelconque sur $\mathrm{AB}$, on pourra la choisir de telle sorte que le tracé des parallèles aux tangentes se fasse le plus commodément possible

(*) Bien entendu, s'il so produit pros des rives des remous importanls, il peul arriver que l'eau remonte en sens inverse du courant principal ct détermine une inflexion du fll.

rans ce cas, le signe négalil de $v$ disparaissant dans $v^{2}$, il faut poser explicitement, pour tous les filets rétrogades:

$$
f=-k d \varepsilon v^{2}
$$

Si l'on introduit cette expression négative dans le calcul, on en conclut que, sur toute la longueur du brin exposé au rellux,

$$
\frac{d^{2} y}{d x^{2}}=+\left(\frac{v}{K}\right)^{2}
$$

Ainsi la théarie s'accorde avec lexperience pour démontrer que lo brin peut toumer sa concavite dans le sens du courant, ct se raccorder inflexionnellement au reste du fil.

(*) C'est la courbe des ponls suspendus, el non pas une chaimelte cav lans l'hypothèse d'une vitesse uniforme, les forces $f$ sont égales, pour des valeurs egales de d $x$, et non pas de ds. 
En pratique, il suffit de transformer quelques points essenticls pour obtenir rapidement la courbe $Y$ '. En particulier le point $\$$, situé sur l'axe AB, s'obtient immédiatement, car il correspond au sommet $\mathrm{S}$ de la courbe $\mathrm{Y}$. De même les points oxtrêmes A' el B', cal ils sont déterminés par les tangentes aux points d'attache $A$ et $B$.

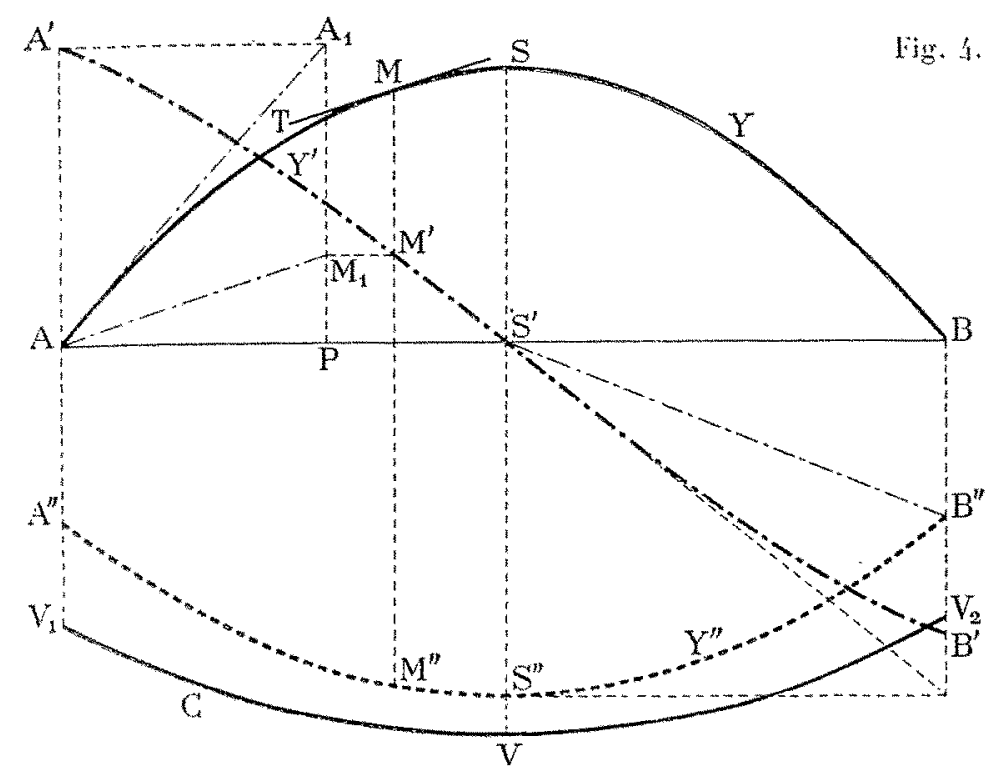

Bien que la tangente soit tracée avec plus ou moins de certitude, les erreurs se compensent d'un point à l'autre et, dans son ensemble, la construction de $\mathrm{Y}^{\prime}$ peut se faire assez cxactement.

En opérant sur $\mathrm{Y}^{\prime}$ comme on vient de le laire pour $\mathrm{Y}$, on peut déduire la courbe dérivée seconde $\mathrm{Y}$ " représentant, en fonction de $x$, le coefficient angulaire $\frac{\mathrm{d}}{\mathrm{d} x}\left(\frac{\mathrm{d} y}{\mathrm{~d} x}\right)$ de la tangonte $\mathrm{X}$ ?.

Dans le cas d'un canal rectiligne, de section symetrique et a l'abri du vent, l'écoulement ost rógulier, et la rópartiLion des vilesses est symétriquo par rapport à l'axo médian SS'. Dans ce cas, les courbes $Y, Y$ ' et $Y$ " sont aussi symétriques par rapport à cet axe (c'est le cas de la figule 4).

Mais, si les conditions précédentes ne sont pas remplies, la répartition des vitesses n'est plus symótrique, et l'ordonnée SS' du point culminant de la courbe $Y$ n'est plus au milieu de $A B$. En outre, le sommet $S$ " de la courbe $Y$ " peut ne plus se trouver sur celte ordonnée SS'; pour's'en rendre compte, il suffit de considérer le cas limite où la vitesse serait maxima en A ou en B. Dans ce cas le sommet S" serait situé sur l'ordonnée limite correspondante, alors que le point culminant du fil se produirait dans l'intervalle $A B$.

Afin de diminuer les risques derreur, il y a intérêt à ce que les diverses courbes soient aussi arquées que possible. pour la courbe $Y$, on réalisera cette condition en dounant au fil une longueur suffisante. Quant aux courbes $Y^{\prime}$ et $Y$ ", si elles ne sont pas naturellement arquées. on amplifiera lócholle de lours ordonnées en prenant une baso AP supérieure à l'unité de longucur; mais il faudra introduire les coefficients d'amplification dans les formules, ce qui n'est d'aucune difficulté.

Ces constructions effectuées, sil'on se reporte à la formule (4), on voit que la courbe $\mathrm{Y}^{3}$ représente égaloment en fonction de $x$ la variation de la quantité $\left(\frac{v}{K}\right)^{2}$. Si donc on réduit les ordonnées à leur racines carrées $\frac{v}{K}$, on obtient une nouvelle courbe $\mathrm{C}$ qui n'est autre, au facteur près, que la courbe figuralive des vilesses.
La surface $S$ de l'aire comprise entre celte courbe $C$ ot l'axe $A B$ sera proportionnelle a la vitesse moyenno cherchée. On a en effet:

$$
S=\int_{0}^{\mathrm{L}} \frac{\eta}{K} \mathrm{~d} x
$$

et, d'autre part:

$$
u=\frac{1}{L} \int_{0}^{\infty} v \mathrm{~d} x
$$

On en conclut, $K$ étant constant:

$$
w=K \frac{S}{L} \text {. }
$$

Il sulfi done de mosurer cetto surface, suivant lun des procédós habituels, pour en déduiro la vilesse moyenne.

Mais ceci suppose cncore qu'on ait fixé la valeur du factour $K$, qui résulle en partie de la constante d'intégration $c$, laissée jusqu'ici arbitraire.

Le principe de cette détermination est le suivant. Si $a$ est lordonnée de la courbe $\mathrm{c}$ aui correspond au filet de vilesse $v$, on a par construction:

$$
a=\frac{v}{K}
$$

Il suffira done de mesurer la vitesse $v$ do l'un des flets, ainsi que lordonnée correspondante $a$ de la courbe $C$, pour en déduire la valeur de $K$. Or, lo relevé expérimental de la vitesse n'est possible simplement que pour la vitesse maxima du courant. Ciost donc cette vitesse $V$ que lon déterminera en pratique. Il lui correspondra lordonnce maxima $A$ de la courbe $C$, et l'on aura:

$$
K=\frac{V}{A}
$$

La mesure de $V$ n'offre aucune difficulté s"il s'agit de la nappe supcrficielle de la rivière.

On abandonne un corps léger au fil le plus rapide du courant, et l'on relève sa vilesso sur un parcours de plusieurs mètres. On reprend celte mosure un cortain nombre de fois, la moyenne des plus fortes valeurs relevées donne très approximativement la vitesse maxima.

Il n'en est plus de même dans le cas duno nappe sous. jacente. Il esi en elfet materiellement impossible de réaliser un flotteur d'uno densité telle qüil se maintionne constamment en suspens au niveau de la nappe expérimontéo.

Nous allons voir que la diffeulte so resout implicitement lorsqu'on applique la méthode du fil a la mesure du débit total de la rivière.

Mesure du débit. - Le debit est domé par la relation:

$$
Q=I I L U
$$

Or la vilesse moyenne $U$ de la rivièro peut-être considérée comme égale à la moyenne des vilesses moyennes $u$ de chacuno des nappes constituantes. si l'on rapporte ces nappes successives à un axe vertical $O z$, on a :

$$
U=\frac{1}{H} \int_{0}^{I I} u \mathrm{~d} s
$$

Mais, en tenant compte des expressions (7) et (8) do $u$ et de $K$,

Par suite:

$$
u=K \frac{S}{L}=\frac{S}{A L} V
$$

$$
U=\frac{1}{H L} \int_{0}^{H} \frac{S}{A} V \mathrm{~d} z
$$


D'oú:

$$
Q=\int_{0}^{\|} \frac{S}{A} V d z
$$

Lorsque l'on peut opérer sut une portion rectiligne d'un cours deau tranquille, et à ccoulement rógulier, la courbe funiculaire reste sensiblement la mòme pour les diverses nappes horizontales. La coincidence est parfaite, surtout si l'on a pu au préalable rectifier le lit et les rives dans la région d'essai. Dans ce cas, le rapport $\frac{S}{A}$ reste constant pour toutes les nappes, et l'on peut écrire:

$$
Q=\frac{S}{A} \int_{0}^{H} V \mathrm{~d} z
$$

ou encore :

$$
Q=H \frac{S}{A} \times \frac{1}{H} \int_{0}^{\mu} V \mathrm{~d}
$$

Mais l'expression $\frac{1}{H} \int_{0}^{I I} V \mathrm{~d} z$ n'est autre quo la moyenne $V_{m}$ des vitesses maxima dans jes différentes nappes, de sorte que le débit $Q$ est finalement domné par l'expression:

$$
Q=H \frac{S}{A} V_{m}
$$

Une expérience simple permet d'ailleurs de déterminer directement la vitesse maxima moyenne $V_{m}$.

Pour mesurer $V_{m}$, on abandonne dans la zone la plus rapide du courant, un flotteur cylindrique de diamètre extérieur aussi uniforme que possible. Ce sera, par exemple, un bàton a parois lisses ou un tube creux. L'une des extrêmités sera lestée d'un poids convenable de manière qu'elle puisse atteindre le fond de la rivière, mais sans le toucher toutefois. L'extrémité supérieure doit à peine émerger au-dessus de la surface libre. de facon à ne pas donner prise á la résistance de l'air ou au vent.

Le flotteúr est entraîné à la dérive et, dès qu'il est en vitesse uniforme, il présente dans le sens du courant une inclinaison constante. Il est alors sollicité sous la même incidence par les filets liquides qui l'entrainent.

Il est d'usage d'en conclure que ces filets actifs communiquent au flotteur une vitesse égale à lá moyenne de leurs vitesses propres, et, conséquemment, un peu supérieure à la vilesse moyenne de la zone. Car le flotteur ne pouvant atteindre exactement le fund de la rivière, les nappes inférieures du courant, c'est-à-dire les moins rapides, ne contribuent pas à son entraînement.

Ce raisonnement est en réalité paradoxal.

Imaginons un flotteur idéal occupant toute la hauteur de l'eau. Sous l'action les filets d'inégales vitesses qui le sollicitent, il prend une vitesse intermédiaire $V$ '. Il s'en suit que les filets $F_{s}$ de vitesse $V s$ supérieure à $V$, exercent sur lo flotteur une poussée accélératice

$$
\int_{\text {Fs }} k\left(V s-V^{\prime}\right)^{2} \mathrm{~d} z
$$

$k$ étant un coefficient linéaire dépendant du diamètre du flotteur, de l'état de sa surface et de la nalure de l'eau (claire ou limoneuse).

Les filets $F_{i}$ de vitesse $V_{i}$ inférieure à $V^{\prime}$ opposent au contraire une résistance

$$
\int_{\mathrm{Fi}} k\left(V_{i}-V^{\prime}\right)^{2} \mathrm{~d} z
$$

Le fiotteur so déplaçant avec une vitesse unforme, la résultante de ces actions opposées est nulle.
On a done :

$$
\begin{aligned}
& \left.\int_{R s}^{2} k V_{s}-V^{\prime}\right)^{2} \mathrm{~d} z=\int_{F i} h\left(V_{i}-V^{\prime}{ }^{2} \mathrm{~d} z\right. \\
& \int_{i s}\left(V_{s}-V^{\prime}\right)^{2} \mathrm{~d} z=\int_{F i}\left(V_{i}-V^{\prime}\right)^{2} \mathrm{~d} z .
\end{aligned}
$$

Cost dalleurs celte relation qui délemine la vitesse de u'anslation $V^{\prime}$ du flotteur.

Si lon effectue le calcul on admettant une variation parabolique de la vitesse des filets,

$$
V=V_{0}+a z-b s^{2}
$$

et pour des valeurs normales des coelficients $a$ et $b$, on trouve que celte vitesse de lranslation $V^{\prime}$ est inférieure a la vitesse moyenne des filets, elle-mème évaluée au moyen de lintégrale:

$$
\frac{1}{H} \int_{0}^{\prime l l} V \mathrm{~d} z \text {. }
$$

lin pratique, linsulfisante longueur du flotteur ayant pour effet de lui communiquel une vitesse de translation supélieure à $V^{\prime}$, on concoit qu il existe une certaine profondeur d'immersion pour laquelle celte vitesse de translation coïncidera avec la vitesse moyenne de la zone.

On trouve $\left(^{*}\right)$ que cette égalité se trouve réalisée, dans des conditions normales d'expérienco, pour uno protondeur dimmersion égale à $0,94 H$.

Or, c'est généralement la dimension adoptéc. Il s'en suit que l'imperfection apparente de l'expérience du flotleur' contribue au contraire à son exactitude.

Il suffira donc de mesurer la vitesse de déplacement du fotteur pour obtenir très approximativement la vitesse maxima moyenne $V_{m}$ du courant: Bien entendu, il faut avoir soin de faire plusieurs mesures successives et de prendre la moyenne des valeurs relevées.
(A suivre.)
H. BurEau,
Licencie ès-sciences Physiques ol Malhemaliques Ingenieur au Laboratoire Central delectricilo

\section{APPLICATIONS DE L'ÉLECTRIGITÉ}

\section{La comande eleotrigue des Machines a PAPIER}

Les moteurs aclionnant les machines à fabriquer le papier, dites machines contimues, doivent répondre à des cxigences lrès particulières. Afin d'en mieux faire ressortir la nature, nous allons d'abord énumérer très brièvement les principaux organes dont se composent ces appareils.

La pâte à papier à l'élat très fluide commence par traverser un épurateur à battements, qui peut être composé de trommels en tóle perforée, animés d'un mouvement de rotalion lenl ef d'un mouvement transversal saccadé, qui rejetlent. les impuretés irop grosses pour Ies traverser.

La pâte est ensuile déversce sur une loile mélallique sans fin, possédant une vitesse d'arancement régulière ct animée en même lemps d'un mouvement transversal allernalif brusque, qui a pour but de faire s'égoutler une certaine partie de l'eau; une autre parlie est aspirće à travers la toile métallique par des botrhes en relalion avec des pompes do dépression.

*) Voir Annales des Ponts-et-Chaussées, 1882,2 semestre, page 69. Voir aussi Flamant. Hydraulique (Encyclopédie des Travaux putblies), dolition 1909 , page 365 . 